\section{BACTERIAL SEPSIS}

\section{Pepducins to the rescue}

Pepducins - specially engineered lipidated peptides that act on the inside cell surface by blocking signalling from G-protein-coupled receptors (GPCRs) - have recently caused a stir in the pharmaceutical world when they showed promising results in the prevention of blood clotting and animal models of breast cancer. Now their engineers have turned to a further disease area - bacterial sepsis. Reporting in Nature Medicine, a team of scientists led by Athan Kuliopulos have developed this new class of compounds into a potential treatment for life-threatening systemic inflammatory response syndrome. These findings are of particular relevance, as, apart from fighting the underlying infection with antibiotics, the treatment of sepsis is currently largely limited to supportive strategies.

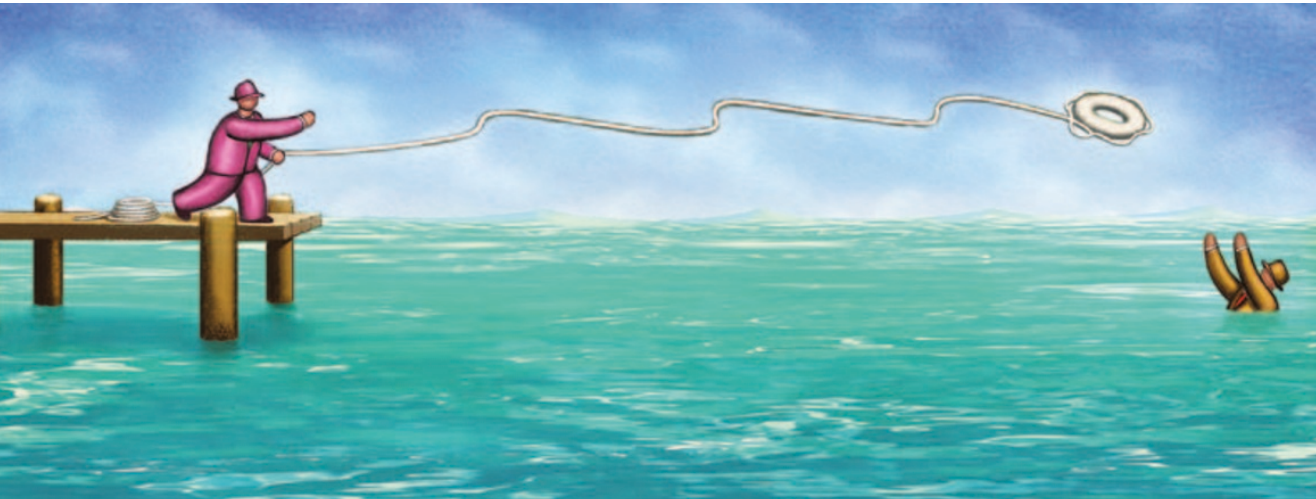

\section{Double trouble}

Two enzymes involved in fatty-acid catabolism are essential for the virulence of Mycobacterium tuberculosis, the agent that causes TB. Isocitrate lyase 1 and 2 (ICL1 and ICL2) are involved in the glyoxylate pathway, which is important in the virulence of many fungal and bacterial pathogens. This makes them attractive targets for the development of broad-spectrum antimicrobials with few side effects, because the glyoxylate pathway is absent in humans.

Microbes use the glyoxylate pathway when growing on fatty-acid substrates, and it is known that genes encoding proteins required for fatty-acid catabolism are upregulated in M. tuberculosis-infected macrophages and mouse lungs. It has also been previously shown that ICL1 is essential for the persistence (chronic phase) of $M$. tuberculosis infection in mice; however, until now, little was known about the role of ICL2, or about carbon utilization pathways important for M. tuberculosis in the lung.
Muñoz-Elías and McKinney report in Nature Medicine that although ICL1 and ICL2 share little sequence similarity, structural features, particularly in the active site loop, suggest that they might have overlapping functions. This is corroborated by the authors' finding that the ability of M. tuberculosis icl mutants to grow on fatty acids in vitro was impaired only when both enzymes were missing.

To determine the contribution of each isoenzyme to virulence, the authors infected mice with mutant mycobacteria lacking one or both ICL isoforms. The absence of ICL1 modestly reduced bacterial load in the lungs and associated tissue pathology during the chronic phase of infection, whereas the absence of ICL2 had no appreciable effect. By contrast, bacteria lacking both enzymes were unable to grow in mice, elicited no pathology and were rapidly cleared from the organs.

Because TB fatality rates are increased in individuals co-infected with HIV, the authors performed similar studies in immunodeficient mice lacking interferon- $\gamma$ (IFN $\gamma$ ) or tumour-necrosis factor- $\alpha$ receptor $(\mathrm{TNF} \alpha \mathrm{R})$. Mycobacteria lacking both ICL isoforms were unable to replicate either in IFN $\gamma$-null or TNF $\alpha$ R-null mice, or in mouse
A crucial player in the pathogenesis of sepsis is the chemokine interleukin 8 (IL-8) - mice deficient for its receptor CXCR2 (a GPCR) are protected from developing sepsis. Kaneider et al. now report the development of pepducins based on the i3 and i1 intracellular loops of CXCR2 and CXCR1 (a second IL-8 receptor in humans), that proved to be extremely powerful in inhibiting IL-8 signalling: in human neutrophils these compounds completely inhibited IL-8-induced calcium responses and migration towards IL- 8 chemotactic gradients.

Using the caecal ligation and puncture (CLP) mouse model of lethal sepsis, the authors demonstrated that the survival rate of mice injected subcutaneously with these pepducins was dramatically enhanced: whereas $17 / 17$ untreated mice died of sepsis, only one of 34 died in the group that received oncedaily injections. Even when this treatment was delayed until 8 hours after the CLP procedure, the survival rate was still $~ 86 \%(26 / 30)$, compared with $0 \%$ in the control group.

Further, the authors demonstrated that the blockade of CXCR1/CXCR2 signalling could reverse several criteria of an established systemic inflammatory response syndrome in mice. The levels of KC, the activated-and non-activated-macrophages. Inhibiting ICL1 and 2 might therefore be a valuable therapeutic strategy for immunocompromised patients.

These findings indicate that an ideal anti-TB drug would be a small molecule that inhibits both ICL1 and 2. No such compound yet exists; however, a prototype inhibitor, 3-nitropropionate (3-NP), which is too toxic to be used in vivo, was used in proof-of-principle in vitro and ex vivo experiments. In media containing different carbon sources, 3-NP blocked the growth of $M$. tuberculosis in the presence of fatty acids, but not carbohydrates. Moreover, addition of 3-NP to M. tuberculosis-infected mouse macrophages prevented intracellular replication in a dose-dependent manner.

The results presented in this study form the basis of a recently launched joint drug discovery programme between the Global Alliance for TB Drug Development and GlaxoSmithKline, which now aims to identify small-molecule inhibitors of these enzymes. Joanna Owens

(D) References and links ORIGINAL RESEARCH PAPER Muñoz-Elías, E. J. \& McKinney, J. D. Mycobacterium tuberculosis isocitrate lyases 1 and 2 are jointly required for in vivo growth and virulence. Nature Med. 15 May 2005 (doi:10.1038/nm1252) 